\title{
ON CLASSICAL FINITE AND AFFINE $\mathcal{W}$-ALGEBRAS
}

\author{
ALBERTO DE SOLE
}

\begin{abstract}
This paper is meant to be a short review and summary of recent results on the structure of finite and affine classical $\mathcal{W}$-algebras, and the application of the latter to the theory of generalized Drinfeld-Sokolov hierarchies.
\end{abstract}

\section{IntRoduction}

In Classical (Hamiltonian) Mechanics the phase space, describing the possible configurations of a physical system, is a Poisson manifold $M$. The physical observables are the smooth functions on $M$ with real values, and they thus form a Poisson algebra (PA). The Hamiltonian equations, describing the time evolution of the system, are written in terms of the Poisson bracket: $\frac{d u}{d t}=\{h, u\}$, where $h(x) \in C^{\infty}(M)$ is the Hamiltonian function (corresponding to the energy observable).

When we quantize a classical mechanic theory we go to Quantum Mechanics. The observables become non commutative objects, and the Poisson bracket is replaced by the commutator of these objects. Hence, the physical observables in quantum mechanics form an associative algebra (AA) $A$. The phase space is then described as a representation $V$ of $A$, and the Schroedinger's equation, describing the evolution of the physical system, is written in terms of this representation: $\frac{d \psi}{d t}=H(\psi)$, where $H \in A$ is the Hamiltonian operator.

Going from a finite to an infinite number of degrees of freedom, we pass from classical and quantum mechanics to classical and quantum field theory respectively. In some sense, the algebraic structure of the space of observables in a conformal field theory is that of a vertex algebra (VA) [Bor86], and its quasi-classical limit is known as Poisson vertex algebra (PVA) [DSK06].

We can summarize the above observations in the following diagram of the algebraic structures of the four fundamental physical theories:

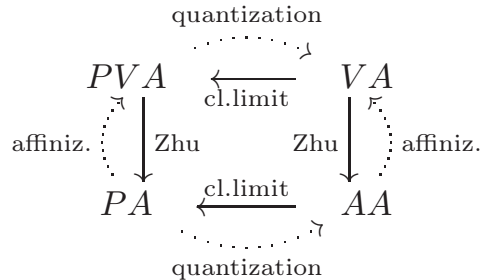

The arrows in the above diagram have the following meaning. If we have a filtered associative algebra, its associated graded is automatically a Poisson algebra called its classical limit. Similarly, if we have a filtered vertex algebra, its associated graded is a Poisson vertex algebra. Furthermore, starting from a positive energy vertex algebra (respectively Poisson vertex algebra) we can construct an associative algebra (resp. Poisson algebra) governing its representation theory, known as its Zhu algebra, [Zhu96]. On the other hand, the processes of going from a classical theory to a quantum theory ("quantization"), or from finitely many to infinitely many degrees of freedom ("affinization"), do not correspond to canonical functors, and they are represented in the diagram with dotted arrows. 
$\mathcal{W}$-algebras provide a very rich family of examples, parametrized by a simple Lie algebra $\mathfrak{g}$ and a nilpotent element $f \in \mathfrak{g}$, which appear in all the 4 fundamental aspects in diagram (0.1):

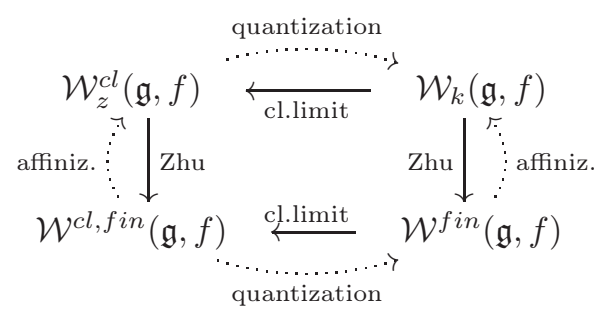

Each of these classes of algebras was introduced and studied separately, with different applications in mind, and only later it became fully clear the relations between them.

Classical finite $\mathcal{W}$-algebras. The classical finite $\mathcal{W}$-algebra $\mathcal{W}^{c l, f i n}(\mathfrak{g}, f)$ is a Poisson algebra, which can be viewed as the algebra of functions on the so-called Slodowy slice $\mathcal{S}(\mathfrak{g}, f)$. It was introduced by Slodowy while studying the singularities associated to the coadjoint nilpotent orbits of $\mathfrak{g}$, [Slo80].

Finite $\mathcal{W}$-algebras. The first appearance of the finite $\mathcal{W}$-algebras $\mathcal{W}^{\text {fin }}(\mathfrak{g}, f)$ was in a paper of Kostant, [Kos78]. He constructed the finite $\mathcal{W}$-algebra for principal nilpotent $f \in \mathfrak{g}$ (in which case it is commutative), and proved that it is isomorphic to the center of the universal enveloping algebra $U(\mathfrak{g})$. The construction was then extended in [Lyn79] for even nilpotent element $f \in \mathfrak{g}$. The general definition of finite $\mathcal{W}$-algebras $\mathcal{W}^{\text {fin }}(\mathfrak{g}, f)$, for an arbitrary nilpotent element $f \in \mathfrak{g}$, appeared much later, [Pre02]. Starting with the work of Premet, there has been a revival of interest in finite $\mathcal{W}$-algebras in connection to geometry and representation theory of simple finite-dimensional Lie algebras, and the theory of primitive ideals (see [Mat90, Pre02, Pre05, BrK06]).

Classical $\mathcal{W}$-algebras. The classical (affine) $\mathcal{W}$-algebras $\mathcal{W}_{z}^{c l}(\mathfrak{g}, f)$ (depending on the parameter $z \in \mathbb{F}$ ) were introduced, for principal nilpotent element $f$, in the seminal paper of Drinfeld and Sokolov [DS85]. They were introduced as Poisson algebras of function on an infinite dimensional Poisson manifold, and they were used to study KdV-type integrable bi-Hamiltonian hierarchies of PDE's, nowdays known as Drinfled Sokolov hierarchies. Subsequently, in the 90's, there was an extensive literature extending the Drinfeld-Sokolv construction of classical $\mathcal{W}$-algebras and the corresponding generalized Drinfeld-Sokolv hierarchies to other nilpotent elements, [dGHM92, FHM92, BdGHM93, DF95, FGMS95, FGMS96]. Only very recently, in [DSKV13a], the classical $\mathcal{W}$-algebras $\mathcal{W}_{z}^{c l}(\mathfrak{g}, f)$ were described as Poisson vertex algebras, and the theory of generalized Drinfeld-Sokolv hierarchies was formalized in a more rigorous and complete way.

$\mathcal{W}$-algebras. The first (quantum affine) $\mathcal{W}$-algebra which appeared in literature was the so called Zamolodchikov $\mathcal{W}_{3}$-algebra [Zam85], which is the $\mathcal{W}$-algebra associated to $\mathfrak{s l}_{3}$ and its principal nilpotent element $f$. It was introduced as a "non-linear" infinite dimensional Lie algebra extending the Virasoro Lie algebra, describing the symmetries of a conformal filed theory. After the work of Zamolodchikov, a number of papers on affine $\mathcal{W}$-algebras appeared in physics literature, mainly as "extended conformal algebras", i.e. vertex algebra extensions of the Virasoro vertex algebra. A review of the subject up to the early 90's may be found in the collection of a large number of reprints on $\mathcal{W}$-algebras [BS95]. The most important results of this period are in the work by Feigin and Frenkel [FF90, FF90b], where the general 
construction of $\mathcal{W}$-algebras, via a quantization of the Drinfeld-Sokolov reduction, was introduced in the case of the principal nilpotent element $f$. For example, if $\mathfrak{g}=s \ell_{n}$, we get the Virasoro vertex algebra for $n=2$, and Zamolodchikov's $\mathcal{W}_{3}$ algebra for $n=3$. The construction was finally generalized to arbitrary nilpotent element $f$ in [KRW03, KW04, KW05]. In these paper, $\mathcal{W}$-algebras were applied to representation theory of superconformal algebras.

A complete understanding of the links among the four different appearances of $\mathcal{W}$-algebras in diagram (0.2) is quite recent. In [GG02] Gan and Ginzburg described the finite $\mathcal{W}$-algebras as a quantization of the Poisson algebra of functions on the Slodowy slice. They thus proved that the classical finite $\mathcal{W}$-algebra $\mathcal{W}^{\text {cl,fin }}(\mathfrak{g}, f)$ can be obtained as the classical limit of the finite $\mathcal{W}$-algebra $\mathcal{W}^{\text {fin }}(\mathfrak{g}, f)$.

As mentioned earlier, the construction of the $\mathcal{W}$-algebra $\mathcal{W}_{k}(\mathfrak{g}, f)$, for principal nilpotent element $f$, due to Feigin and Frenkel [FF90], was obtained as a "quantization" of the Drinfeld-Sokolov construction of the classical $\mathcal{W}$-algebra $\mathcal{W}_{z}^{c l}(\mathfrak{g}, f)$. But it is only in [DSKV13a] that the classical $\mathcal{W}$-algebra $\mathcal{W}_{z}^{c l}(\mathfrak{g}, f)$ is described as a Poisson vertex algebra which can be obtained as classical limit of the $\mathcal{W}$-algebra $\mathcal{W}_{k}(\mathfrak{g}, f)$.

Furthermore, in [DSK06] it is proved there that the ( $H$-twisted) Zhu algebra $Z h u_{H} \mathcal{W}_{k}(\mathfrak{g}, f)$ is isomorphic to the corresponding finite $\mathcal{W}$-algebra $\mathcal{W}^{\text {fin }}(\mathfrak{g}, f)$. Hence, their categories of irreducible representations are equivalent. (This result was independently proved in [Ara07] for principal nilpotent $f$.) A similar result for classical $\mathcal{W}$-algebras holds as well. It is also proved in the Appendix of [DSK06] (in collaboration with A. D'Andrea, C. De Concini and R. Heluani) that the quantum Hamiltonian reduction definition of finite $\mathcal{W}$-algebras is equivalent to the definition via the Whittaker models, which goes back to [Kos78].

In the present paper we describe in more detail the "classical" part of diagram (0.2): in Section 1 we describe the Poisson structure of the Slodowy slice and we introduce the classical finite $\mathcal{W}$-algebra $\mathcal{W}^{\text {cl, fin }}(\mathfrak{g}, f)$. In order to describe the affine analogue of it, we first need to describe the classical finite $\mathcal{W}$-algebra $\mathcal{W}^{\text {cl, fin }}(\mathfrak{g}, f)$ as a Hamiltonian reduction, which is done in Section 1.4. By taking the affine analogue of this construction, we obtain the classical $\mathcal{W}$-algebra $\mathcal{W}_{z}^{c l}(\mathfrak{g}, f)$. Finally, in Section 2.5 we describe, following [DSKV13a], how classical $\mathcal{W}$-algebras are used to study the generalized Drinfeld-Sokolov bi-Hamiltonian hierarchies.

The present paper is based on lectures given by the author for the conference Lie superalgberas, at INdAM, Roma, Italy, in December 2012, and for the conference Symmetries in Mathematics and Physics, at IMPA, Rio de Janeiro, Brazil, in June 2013.

\section{Classical finite $\mathcal{W}$-algebras}

1.1. Poisson manifolds. Recall that, by definition, a Poisson manifold is a manifold $M=M^{n}$ together with a Poisson bracket $\{\cdot, \cdot\}$ on the algebra of functions $C^{\infty}(M)$, making it a Poisson algebra By the Leibniz rule, we can write the Poisson bracket as

$$
\{f(x), g(x)\}=\sum_{i, j} K_{i j}(x) \frac{\partial f}{\partial x_{i}} \frac{\partial g}{\partial x_{j}} .
$$

The bivector $\eta=\sum_{i, j} K_{i j}(x) \frac{\partial}{\partial x_{i}} \wedge \frac{\partial}{\partial x_{j}} \in \Gamma\left(\bigwedge^{2} T M\right)$ is the Poisson structure of the manifold. To every function $h \in C^{\infty}(M)$ on a Poisson manifold $M$ we associate a Hamiltonian vector field $X_{h}=\sum_{i, j=1}^{n} K(x)_{i j} \frac{\partial h(x)}{\partial x_{i}} \frac{\partial}{\partial x_{j}}=\{h, \cdot\}$, and the 
corresponding Hamiltonian flow (or evolution):

$$
\frac{d x}{d t}=\{h, x\}=K(x) \nabla_{x} h .
$$

(This is the Hamiltonian equation associated to the Hamiltonian function $h$.) If we start from a point $x \in M$ and we follow all the possible Hamiltonian flows (1.1) thorough $x$, we cover the symplectic leaf through $x$. The Poisson manifold $M$ is then disjoint union of its symplectic leaves: $M=\sqcup_{\alpha} S_{\alpha}$ (which are symplectic manifolds).

It is natural to ask when a Poisson structure $\eta$ on a Poisson manifold $M$ induces a Poisson structure on a submanifold $N$. Some sufficient condition is given by the following

Proposition 1.1 (Va94). Suppose that, for every point $x \in N$, denoting by $(S, \omega)$ the symplectic leave of $M$ through $x$, we have

(i) the restriction of the symplectic form $\omega(x)$ of $T_{x} S$ to $T_{x} N \cap T_{x} S$ is nondegenerate;

(ii) $N$ is transverse to $S$, i.e. $T_{x} N+T_{x} S=T_{x} M$.

Then, the Poisson structure on $M$ naturally induces a Poisson structure on $N$, and the symplectic leave of $N$ through $x$ is $N \cap S$.

If $\mathfrak{g}$ be a Lie algebra, the dual space $\mathfrak{g}^{*}$ has a natural structure of a Poisson manifold. Indeed, the Lie bracket $[\cdot, \cdot \cdot]$ on $\mathfrak{g}$ extends uniquely to a Poisson bracket on the symmetric algebra $S(\mathfrak{g})$ : if $\left\{x_{i}\right\}_{i=1}^{n}$ is a basis of $\mathfrak{g}$, we have

$$
\{P, Q\}=\sum_{i, j=1}^{n} \frac{\partial P}{\partial x_{i}} \frac{\partial Q}{\partial x_{j}}\left[x_{i}, x_{j}\right] .
$$

We can think at $S(g)$ as the algebra of polynomial functions on $\mathfrak{g}^{*}$. Hence, $\mathfrak{g}^{*}$ has an induced structure of a Poisson manifold. In coordinates, if we think at $\left\{x_{i}\right\}_{i=1}^{n}$ as linear functions, or local coordinates, on $\mathfrak{g}^{*}$, and we let $\left\{\xi_{i}=\frac{\partial}{\partial x_{i}}\right\}_{i=1}^{n}$ be the dual basis of $\mathfrak{g}^{*}$, then, by (1.2), the Poisson structure $\eta \in \Gamma\left(\bigwedge^{2} T \mathfrak{g}^{*}\right)$ evaluated at $\xi \in \mathfrak{g}^{*}$ is

$$
\eta(\xi)=\sum_{i, j=1}^{n} \xi\left(\left[x_{i}, x_{j}\right]\right) \xi_{i} \wedge \xi_{j}=\sum_{j=1}^{n} \operatorname{ad}^{*}\left(x_{j}\right)(\xi) \wedge \xi_{j} \in \wedge^{2}\left(\mathfrak{g}^{*}\right) .
$$

By (1.3), the Hamiltonian vector field associated to $a \in \mathfrak{g}$ is ad* $a$, and the corresponding Hamiltonian flow through $\xi \in \mathfrak{g}^{*}$ is $\operatorname{Ad}^{*}\left(e^{t a}\right)(\xi)$. Hence, the symplectic leaves of $\mathfrak{g}^{*}$ are the coadjoint orbits $S=\operatorname{Ad}^{*} G(\xi)$ where $G$ is the connected Lie group with Lie algebra $\mathfrak{g}$. The Poisson structure on the coadjoint orbits $\operatorname{Ad}^{*} G(\xi)$ is known as Kirillov-Kostant Poisson structure. Its inverse is a symplectic structure. At the point $\xi \in \mathfrak{g}^{*}$ it is the following non-degenerate skewsymmetric form $\omega(\xi)$ on $\operatorname{ad}^{*} \mathfrak{g}(\xi)$ :

$$
\omega(\xi)\left(\operatorname{ad}^{*}(a)(\xi), \operatorname{ad}^{*}(b)(\xi)\right)=\xi([a, b]) .
$$

1.2. The Poisson structure on the Slodowy slice. Let $\mathfrak{g}$ be a reductive finite dimensional Lie algebra, and let $f \in \mathfrak{g}$ be a nilpotent element. By the JacobosonMorozov Theorem, $f$ can be included in an $\mathfrak{s l}_{2}$-triple $\{e, h=2 x, f\}$, see e.g. [CM93]. Let $(\cdot \mid \cdot)$ be a non-degenerated invariant symmetric bilinear form on $\mathfrak{g}$, and let $\Phi: \mathfrak{g} \stackrel{\sim}{\rightarrow} \mathfrak{g}^{*}$ be the isomorphism associated to this bilinear form: $\Phi(a)=(a \mid \cdot)$. We also let $\chi=\Phi(f)=(f \mid \cdot) \in \mathfrak{g}^{*}$.

The Slodowy slice [Slo80] associated to this $\mathfrak{s l}_{2}$-triple element is, by definition, the following affine space

$$
\mathcal{S}=\Phi\left(f+\mathfrak{g}^{e}\right)=\left\{\begin{array}{c}
\chi \chi \\
4
\end{array}(a) \mid a \in \mathfrak{g}^{e}\right\} \subset \mathfrak{g}^{*} .
$$


Let $\xi=\Phi(f+r), r \in \mathfrak{g}^{e}$, be a given point of the Slodowy slice. The tangent space to the coadjoint orbit $\mathrm{Ad}^{*} G(\xi)$ at $\xi$ is $T_{\xi}\left(\operatorname{Ad}^{*} G(\xi)\right)=\operatorname{ad}^{*}(\mathfrak{g})(\xi)=\Phi([f+r, \mathfrak{g}])$, while the tangent space to the Slodowy slice at $\xi$ is $T_{\xi}(\mathcal{S}) \simeq \Phi\left(\mathfrak{g}^{e}\right)$. Recalling (1.4), one can check that the assumptions of Proposition 1.1 hold, [GG02]:

(i) The restriction of the symplectic form (1.4) to $T_{\xi}\left(\operatorname{Ad}^{*} G(\xi)\right) \cap T_{\xi}(\mathcal{S})$ is nondegenerate. In other words, if $a \in \mathfrak{g}$ is such that $[f+r, a] \in \mathfrak{g}^{e}$ and $a \perp$ $[f+r, \mathfrak{g}] \cap \mathfrak{g}^{e}$, then $a=0$.

(ii) The Slodowy slice $\mathcal{S}$ intersect transversally the coadjoint orbit at $\xi$, i.e. $[f+$ $r, \mathfrak{g}]+\mathfrak{g}^{e}=\mathfrak{g}$.

It then follows by Proposition 1.1 that $\mathcal{S} \subset \mathfrak{g}^{*}$ is a Poisson submanifold, i.e. it has a Poisson structure induced by the Kirillov-Kostant structure on $\mathfrak{g}^{*}$.

Definition 1.2. The classical finite $\mathcal{W}$ algebra $\mathcal{W}^{c l, f i n}(\mathfrak{g}, f) \simeq S\left(\mathfrak{g}^{f}\right)$ is the algebra of polynomial functions on the Slodowy slice $\mathcal{S}$

Clearly, the dual space to $\Phi\left(\mathfrak{g}^{e}\right)$ is $\mathfrak{g}^{f}$. Hence, by the definition (1.5) of $\mathcal{S}$, we can identify $\mathcal{W}^{c l, f i n}(\mathfrak{g}, f)$, as a polynomial algebra, with the symmetric algebra over $\mathfrak{g}^{f}$. In fact, we can write down an explicit formula for the Poisson bracket of the classical finite $\mathcal{W}$-algebra. We have the direct sum decomposition: $\mathfrak{g}=[e, \mathfrak{g}] \oplus \mathfrak{g}^{f}$, and, for $a \in \mathfrak{g}$, we denote $a^{\sharp}$ its projection on $\mathfrak{g}^{f}$. Let $\left\{q_{i}\right\}_{i=1}^{k}$ be a basis of $\mathfrak{g}^{f}$ consisting of ad $x$-eigenvectors, and let $\left\{q^{i}\right\}_{i=1}^{k}$ be the dual basis of $\mathfrak{g}^{e}$. For $i \in\{1, \ldots, k\}$, we let $\delta(i) \in \frac{1}{2} \mathbb{Z}$ be ad $x$-eigenvalue of $q^{i}$. By representation theory of $\mathfrak{s l}_{2}$, a basis of $\mathfrak{g}$ is

$$
\left\{q_{n}^{i}:=(\operatorname{ad} f)^{n} q^{i} \mid n=0, \ldots, 2 \delta(i), i=1, \ldots, k\right\},
$$

and let $\left\{q_{i}^{n} \mid n=0, \ldots, 2 \delta(i), i=1, \ldots, k\right\}$, be the dual basis of $\mathfrak{g}$. (Here and further we let $q_{0}^{i}=q^{i}$ and $q_{i}^{0}=q^{i}$.)

Theorem 1.3 ([DSK13c]). The Poisson bracket on the classical finite $\mathcal{W}$-algebra $\mathcal{W}^{c l, f i n}(\mathfrak{g}, f)$ is $\left(p, q \in \mathfrak{g}^{f}\right)$ :

$$
\{p, q\}_{\mathcal{S}}=[p, q]+\sum_{s=1}^{\infty} \sum_{i_{1}, \ldots, i_{s}=1}^{k} \sum_{m_{1}, \ldots, m_{s}=0}^{d}\left[p, q_{m_{1}}^{i_{1}}\right]^{\sharp}\left[q_{i_{1}}^{m_{1}+1}, q_{m_{2}}^{i_{2}}\right]^{\sharp} \ldots\left[q_{i_{s}}^{m_{s}+1}, q\right]^{\sharp} .
$$

Example 1.4. If $q \in \mathfrak{g}_{0}^{f}=\mathfrak{g}_{0}^{e}$, then $\left[q_{i}^{m+1}, q\right]^{\sharp}=0$ for all $i, m$. Hence, $\{p, q\}_{\mathcal{S}}=$ $[p, q] \in \mathfrak{g}^{f}$.

1.3. Classical Hamiltonian reduction. In order to define, in Section 2, the classical $\mathcal{W}$-algebra $\mathcal{W}_{z}^{c l}(\mathfrak{g}, f)$, i.e. affine analogue of the classical finite $\mathcal{W}$-algebra $\mathcal{W}^{\text {cl,fin }}(\mathfrak{g}, f)$, it is convenient to describe the Poisson structure on the Slodowy slice $\mathcal{S}$ via a Hamiltonian reduction of $\mathfrak{g}^{*}$. In this section we describe, in a purely algebraic setting, the general construction of the classical Hamiltonian reduction of a Hamiltonian action of a Lie group $N$ on a Poisson manifold $P$. In the next Section 1.4 we then describe the classical finite $\mathcal{W}$-algebra $\mathcal{W}^{c l, f i n}(\mathfrak{g}, f)$ as a Hamiltonian reduction.

Recall that the classical Hamiltonian reduction is associated to a Poisson manifold $M$, a Lie group $N$ with a Hamiltonian action on $M$, and a submanifold $\mathcal{O} \subset \mathfrak{n}^{*}$ which is invariant by the coadjoint action of $N$. The corresponding Hamiltonian reduction is, by definition, $\mu^{-1}(\mathcal{O}) / N$, where $\mu: M \rightarrow \mathfrak{n}^{*}$ is the moment map associated to the Hamiltonian action of $N$ on $M$. One shows that, indeed, $\mu^{-1}(\mathcal{O}) / N$ has a Poisson structure induced by that to $M$, [GS90].

On a purely algebraic level, going to the algebras of functions, the classical Hamiltonian reduction can be defined as follows. Let $(P, \cdot,\{\cdot, \cdot\})$ be a unital Poisson algebra. Let $\mathfrak{n}$ be a Lie algebra. Let $\phi: \mathfrak{n} \rightarrow P$ be a Lie algebra homomorphism, 
and denote by $\phi: S(\mathfrak{n}) \rightarrow P$ the corresponding Poisson algebra homomorphism. Let $I \subset S(\mathfrak{n})$ be a subset which is invariant by the adjoint action of $\mathfrak{n}$, i.e. such that $\operatorname{ad}(\mathfrak{n})(I) \subset I$. Consider the ideal $P \phi(I)$ of $P$ generated by $\phi(I)$. Note that, in general, $P \phi(I)$ is NOT a Poisson ideal, so the quotient space $P / P \phi(I)$ has an induced structure of a commutative associative algebra, but NOT of a Poisson algebra.

Definition 1.5. The Hamiltonian reduction of the Poisson algebra $P$ associated to the Lie algebra homomorphism $\phi: \mathfrak{n} \rightarrow P$ and to the ad $\mathfrak{n}$-invariant subset $I \subset S(\mathfrak{n})$ is, as a space,

$$
\mathcal{W}(P, \mathfrak{n}, I):=(P / P \phi(I))^{\mathfrak{n}}=\{f \in P \mid\{\phi(\mathfrak{n}), f\} \subset P \phi(I)\} / P \phi(I) .
$$

Proposition 1.6. The Hamiltonian reduction $\mathcal{W}(P, \mathfrak{n}, I)$ has an induced structure of a Poisson algebra.

Proof. First, it follows by the Leibniz rule that $\{f \in P \mid\{\phi(\mathfrak{n}), f\} \subset P \phi(I)\} \subset P$ is a subalgebra with respect to the commutative associative product of $P$, and, since by assumption the set $I$ is ad(n)-invariant, $P \phi(I) \subset\{f \in P \mid\{\phi(\mathfrak{n}), f\} \subset P \phi(I)\}$ is its ideal. Hence, their quotient $W(P, \mathfrak{n}, S)$ has an induced commutative associative product. We use the same argument for the Poisson structure: we claim that

(i) $\{f \in P \mid\{\phi(\mathfrak{n}), f\} \subset P \phi(I)\} \subset P \phi(I)$ is a Lie subalgebra,

(ii) and that $P \phi(I) \subset\{f \in P \mid\{\phi(\mathfrak{n}), f\} \subset P \phi(I)\}$ is its Lie algebra ideal.

Suppose that $f, g \in P$ are such that $(\operatorname{ad} \phi(\mathfrak{n}))(f) \subset P \phi(I)$ and $(\operatorname{ad} \phi(\mathfrak{n}))(g) \subset$ $P \phi(I)$. Then, by the Jacobi identity,

$$
\begin{aligned}
& \{\phi(\mathfrak{n}),\{f, g\}\} \subset\{\{\phi(\mathfrak{n}), f\}, g\}+\{f,\{\phi(\mathfrak{n}), g\}\} \subset\{P \phi(I), g\}+\{f, P \phi(I)\} \\
& \subset P\{\phi(I), g\}+P\{f, \phi(I)\}+P \phi(I) \subset P\{\phi(S(\mathfrak{n})), g\}+P\{\phi(S(\mathfrak{n})), f\}+P \phi(I) \\
& \subset P\{\phi(n), g\}+P\{\phi(\mathfrak{n}), f\}+P \phi(I) \subset P \phi(I) .
\end{aligned}
$$

In the second inclusion we used the assumption on $f$ and $g$, in the third inclusion we used the Leibniz rules, in the fourth inclusion we used the fact that, by construction, $I \subset S(\mathfrak{n})$, in the fifth inclusion we used the Leibniz rules, and in the last inclusion we used again the assumption on $f$ and $g$. This proves claim (i).

For claim (ii), let $f \in P$ be such that $\{\phi(\mathfrak{n}), f\} \subset P \phi(I)$. We have, with the same line of arguments as above,

$$
\begin{aligned}
& \{P \phi(I), f\} \subset P\{\phi(I), f\}+P \phi(I) \subset P\{\phi(S(\mathfrak{n})), f\}+P \phi(I) \\
& \subset P\{\phi(\mathfrak{n}), f\}+P \phi(I) \subset P \phi(I) .
\end{aligned}
$$

1.4. The Slodowy slice via Hamiltonian reduction. We want to describe the classical finite $\mathcal{W}$-algebra $\mathcal{W}^{c l, f i n}(\mathfrak{g}, f)$ introduced in Section 1.2 as a Hamiltonian reduction of the Poisson algebra $S(\mathfrak{g})$.

We have the ad $x$-eignespace decomposition $\mathfrak{g}=\bigoplus_{i \in \frac{1}{2} \mathbb{Z}} \mathfrak{g}_{i}$. Let $\omega$ be the following non-degenerate skewsymmetric bilinear form on $\mathfrak{g}_{\frac{1}{2}}$ :

$$
\omega(u, v)=(f \mid[u, v]) .
$$

Let $\ell \subset \mathfrak{g}_{\frac{1}{2}}$ be a maximal isotropic subspace. Consider the nilpotent subalgebra

$$
\mathfrak{n}=\ell \oplus \mathfrak{g}_{\geq 1} \subset \mathfrak{g} .
$$

Since $\ell \subset \mathfrak{g}_{\frac{1}{2}}$ is isotropic w.r.t. the bilinear form $(1.8)$, we have $(f \mid[\mathfrak{n}, \mathfrak{n}])=0$. Hence, the subset

$$
I=\{n-(f \mid n) \mid n \in \mathfrak{n}\} \subset S(\mathfrak{n}),
$$


is invariant by the adjoint action of $\mathfrak{n}$. Hence, we can consider the corresponding Hamiltonian reduction (1.7) applied to the data $(S(\mathfrak{g}), \mathfrak{n}, I)$.

Theorem 1.7 ([DSK13c]). The classical finite $\mathcal{W}$-algebra $\mathcal{W}^{\text {cl,fin }}(\mathfrak{g}, f)$ is isomorphic to the Hamiltonian reduction of the Poisson algebra $S(\mathfrak{g})$, associated to the Lie algebra $\mathfrak{n} \subset S(\mathfrak{g})$ given by (1.9), and the ad $\mathfrak{n}$-invariant subset $I \subset S(\mathfrak{n})$ in (1.10):

$$
\begin{aligned}
& \mathcal{W}^{c l, f i n}(\mathfrak{g}, f) \simeq \mathcal{W}(S(\mathfrak{g}), \mathfrak{n}, I) \\
& =\left\{p \in S(\mathfrak{g}) \mid\{\mathfrak{n}, p\} \subset\langle n-(f \mid n)\rangle_{n \in \mathfrak{n}}\right\} / S(\mathfrak{g})\langle n-(f \mid n)\rangle_{n \in \mathfrak{n}}
\end{aligned}
$$

In geometric terms, the restriction map $\mu: \mathfrak{g}^{*} \rightarrow \mathfrak{n}^{*}$ is a map of Poisson manifolds (the moment map), and the corresponding dual map $\mu^{*}: \mathfrak{n} \rightarrow \mathfrak{g}$, is the inclusion map. The element $\chi=\left.(f \mid \cdot)\right|_{\mathfrak{n}} \in \mathfrak{n}^{*}$ is a character of $\mathfrak{n}$, in the sense that $\chi([\mathfrak{n}, \mathfrak{n}])=0$ (by the assumption that $\ell$ is maximal isotropic). Hence, $\chi$ is fixed by the coadjoint action of $N$, the Lie group of $\mathfrak{n}$. We can then consider the Hamiltonian reduction of the Poisson manifold $\mathfrak{g}^{*}$, by the Hamiltonian action of $N$ on $\mathfrak{g}^{*}$ given by the moment map $\mu$, associated to the $N$-fixed point $\chi \in \mathfrak{g}^{*}$ :

$$
\text { Ham.Red. }\left(\mathfrak{g}^{*}, N, \chi\right)=\mu^{-1}(\chi) / N=\Phi\left(f+\mathfrak{n}^{\perp}\right) / N \text {. }
$$

Theorem 1.7 can be then viewed as the algebraic analogue of the following result of Gan and Ginzburg:

Theorem 1.8 ([GG02]). The coadjoint action $N \times \mathcal{S} \rightarrow \Phi\left(f+\mathfrak{n}^{\perp}\right)$ is an isomorphism of affine varieties. The corresponding bijection

$$
\mathcal{S} \simeq \mu^{-1}(\chi) / N=\text { Ham.Red. }\left(\mathfrak{g}^{*}, N, \chi\right),
$$

is an isomorphism of Poisson manifolds.

\section{Classical $W$-algebras}

2.1. Poisson vertex algebras. In this section we introduce the notions of Lie conformal algebra and of Poisson vertex algebra. They are, in some sense, the "affine analogue" of a Lie algebra and of a Poisson algebra respectively.

Definition 2.1. A Lie conformal algebra is a $\mathbb{F}[\partial]$-module $R$ with a bilinear $\lambda$ bracket $\left[\cdot{ }_{\lambda} \cdot\right]: R \times R \rightarrow \mathbb{F}[\lambda] \otimes R$ satisfying the following axioms:

(i) sesquilinearity: $\left[\partial a_{\lambda} b\right]=-\lambda\left[a_{\lambda} b\right],\left[a_{\lambda} \partial b\right]=(\partial+\lambda)\left[a_{\lambda} b\right]$;

(ii) skewsymmetry: $\left[a_{\lambda} b\right]=-\left[b_{-\lambda-\partial} a\right]$ (where $\partial$ is moved to the left);

(iii) Jacobi identity: $\left[a_{\lambda}\left[b_{\mu} c\right]\right]-\left[b_{\mu}\left[a_{\lambda} c\right]\right]=\left[\left[a_{\lambda} b\right]_{\lambda+\mu} c\right]$.

Example 2.2. Let $\mathfrak{g}$ be a Lie algebra with a symmetric invariant bilinear form $(\cdot \mid \cdot)$. The corresponding current Lie conformal algebra is, by definition, $R=\mathbb{F}[\partial] \mathfrak{g} \oplus \mathbb{F}$, with $\lambda$-bracket $(s$ is a fixed element of $\mathfrak{g})$ :

$$
\left[a_{\lambda} b\right]=[a, b]+(a \mid b) \lambda+z(s \mid[a, b]),
$$

for $a, b \in \mathfrak{g}$, extended to $R$ by saying that $\mathbb{F}$ is central, and by sesquilinearity. (The term $(a \mid b) \lambda$ is a 2 -cocycle, defining a central extension, and $z(s \mid[a, b])$ is a trivial 2-cocycle.)

Definition 2.3. A Poisson vertex algebra is a commutative associative differential algebra $\mathcal{V}$ (with derivation $\partial$ ) endowed with a Lie conformal algebra $\lambda$-bracket $\{\cdot \lambda \cdot\}$, satisfying the Leibniz rule

$$
\left\{a_{\lambda} b c\right\}=\left\{a_{\lambda} b\right\} c+\left\{a_{\lambda} c\right\} b .
$$

Note that by the skewsymmetry axiom and the left Leibniz rule (2.2) we get the right Leibniz rule:

$$
\left\{a b_{\lambda} c\right\}=\left\{a_{\lambda+\partial} c\right\} \rightarrow b+\left\{b_{\lambda+\partial} c\right\} \rightarrow a,
$$

where the arrow means that $\partial$ should be moved to the right. 
Example 2.4. If $R$ is a Lie conformal algebra, then $S(R)$ has a natural structure of a Poisson vertex algebra, with $\lambda$-bracket extending the one in $R$ by the left and right Leibniz rules.

Example 2.5. As a special case of Example 2.4, consider the current Lie conformal algebra $R=\mathbb{F}[\partial] \mathfrak{g} \oplus \mathbb{F}$ associated to the Lie algebra $\mathfrak{g}$ and the symmetric invariant bilinear form $(\cdot, \cdot)$, defined in Example 2.2. The affine Poisson vertex algebra is, by definition, $\mathcal{V}_{z}(\mathfrak{g})=S(\mathbb{F}[\partial] \mathfrak{g})$, with $\lambda$-bracket $(2.1)$, extended by sesquilinearity and Leibniz rules. If $\left\{u_{i}\right\}_{i=1}^{n}$ is a basis of $\mathfrak{g}$, the general formula for the $\lambda$-bracket is:

$$
\begin{aligned}
& \left\{P_{\lambda} Q\right\}_{z}=\sum_{i, j=1}^{n} \sum_{m, n \in \mathbb{Z}_{+}} \frac{\partial Q}{\partial u_{j}^{(n)}}(\lambda+\partial)^{n} \\
& \left(\left[u_{i}, u_{j}\right]+\left(u_{i} \mid u_{j}\right)(\lambda+\partial)+z\left(s \mid\left[u_{i}, u_{j}\right]\right)\right)(-\lambda-\partial)^{m} \frac{\partial Q}{\partial u_{j}^{(n)}}(\lambda+\partial)^{n} .
\end{aligned}
$$

This is the "affine analogue" of the usual Poisson algebra structure on $S(\mathfrak{g})$. It is a 1-parameter family of Poissson vertex algebras, depending on the parameter $z \in \mathbb{F}$. (Having a 1-parameter family is important for applications to the theory of integrable systems.)

2.2. Hamiltonian reduction of Poisson vertex algebras. The classical Hamiltonian reduction construction described in Section 1.3 has an "affine analogue" for Poisson vertex algebras.

Let $\left(\mathcal{V}, \partial, \cdot,\left\{\cdot_{\lambda} \cdot\right\}\right)$ be a Poisson vertex algebra. Let $R$ be a Lie conformal algebra. Let $\phi: R \rightarrow \mathcal{V}$ be a Lie conformal algebra homomorphism, which we extend to a Poisson vertex algebra homomorphism $\phi: S(R) \rightarrow \mathcal{V}$. Let $I \subset S(R)$ be a subset which is invariant by the adjoint action of $R$, i.e. such that $\left[R_{\lambda} I\right] \subset \mathbb{F}[\lambda] \otimes I$. Consider the differential algebra ideal $\langle\phi(I)\rangle_{\mathcal{V}} \subset \mathcal{V}$ of $\mathcal{V}$ generated by $\phi(I)$. The quotient space $\mathcal{V} /\langle\phi(I)\rangle_{\mathcal{V}}$ has an induced structure of a (commutative associative) differential algebra (but NOT of a Poisson vertex algebra).

Definition 2.6. The Hamiltonian reduction of the Poisson vertex algebra $\mathcal{V}$ associated to the Lie conformal algebra homomorphism $\phi: R \rightarrow \mathcal{V}$ and to the $R$-invariant subset $I \subset S(R)$ is, as a space,

$$
\begin{aligned}
& \mathcal{W}(\mathcal{V}, R, I):=\left(\mathcal{V} /\langle\phi(I)\rangle_{\mathcal{V}}\right)^{R} \\
& =\left\{f \in \mathcal{V} \mid\left\{\phi(a)_{\lambda} f\right\} \in \mathbb{F}[\lambda] \otimes\langle\phi(I)\rangle_{\mathcal{V}} \forall a \in R\right\} /\langle\phi(I)\rangle_{\mathcal{V}} .
\end{aligned}
$$

Proposition 2.7. The Hamiltonian reduction $\mathcal{W}(\mathcal{V}, R, I)$ has an induced structure of a Poisson vertex algebra.

Proof. It is analogue to the proof of Proposition 1.6.

2.3. Construction of the classical $\mathcal{W}$-algebras. In analogy with the construction, in Theorem 1.7, of the classical finite $\mathcal{W}$-algebra $\mathcal{W}^{c l, f i n}(\mathfrak{g}, f)$ via Hamiltonian reduction, we define its "affine analogue", the classical $\mathcal{W}$-algebra $\mathcal{W}_{z}^{c l}(\mathfrak{g}, f)$, via a Hamiltonian reduction of the affine Poisson vertex algebra $\mathcal{V}_{z}(\mathfrak{g})$ in Example 2.5.

Let, as in Section $1.4, \mathfrak{g}$ be a reductive finite dimensional Lie algebra, with a non-degenerate symmetric bilinear form $(\cdot \mid \cdot)$. Let $(e, h=2 x, f)$ be an $\mathfrak{s l}_{2}$-triple in $\mathfrak{g}$, and consider the ad $x$-eignespace decomposition $\mathfrak{g}=\bigoplus_{i \in \frac{1}{2} \mathbb{Z}} \mathfrak{g}_{i}$, Recall the definitions of the bilinear form $\omega$ on $\mathfrak{g}_{\frac{1}{2}}$ in (1.8), of the nilpotent subalgebra $\mathfrak{n} \subset \mathfrak{g}$ in (1.9), and of the ad $\mathfrak{n}$-invariant subset $I=\{n-(f \mid n)\}_{n \in \mathfrak{n}} \subset S(\mathfrak{n})$, as in (1.10). Consider the current Lie conformal algebra $\mathbb{F}[\partial] \mathfrak{n}$ with $\lambda$-bracket $\left\{a_{\lambda} b\right\}=[a, b]$ for $a, b \in \mathfrak{n}$, and extended to $R$ by sesquilinearity. It is obviously a Lie conformal subalgebra of $\mathcal{V}_{z}(\mathfrak{g})$. Hence, the inclusion $S(\mathbb{F}[\partial] \mathfrak{n}) \subset \mathcal{V}_{z}(\mathfrak{g})$ is a homomorphism 
of Poisson vertex algebras. Furthermore, the set $I=\{n-(f \mid n)\}_{n \in \mathfrak{n}} \subset S(\mathbb{F}[\partial] \mathfrak{n})$ is invariant by the $\lambda$-adjoint action of $\mathbb{F}[\partial] \mathfrak{n}$. Let $\langle n-(f \mid n)\rangle_{n \in \mathfrak{n}} \subset \mathcal{V}_{z}(\mathfrak{g})$ be the differential algebra ideal of $\mathcal{V}_{z}(\mathfrak{g})$ generated by $I=\{n-(f \mid n)\}_{n \in \mathfrak{n}}$. We can consider the Hamiltonian reduction associated to the data $\left(\mathcal{V}_{z}(\mathfrak{g}, f), \mathbb{F}[\partial] \mathfrak{n}, I\right)$.

Definition 2.8 ([DSKV13a]). The classical $\mathcal{W}$-algebra is the following 1-parameter family (parametrized by $z \in \mathbb{F}$ ) of Poisson vertex algebras

$$
\begin{aligned}
& \mathcal{W}_{z}^{c l}(\mathfrak{g}, f) \simeq \mathcal{W}\left(\mathcal{V}_{z}(\mathfrak{g}), \mathbb{F}[\partial] \mathfrak{n}, I\right) \\
& =\left\{P \in S(\mathbb{F}[\partial] \mathfrak{g}) \mid\left\{a_{\lambda} P\right\}_{z} \in \mathbb{F}[\lambda] \otimes\langle n-(f \mid n)\rangle_{n \in \mathfrak{n}} \forall a \in \mathfrak{n}\right\} /\langle n-(f \mid n)\rangle_{n \in \mathfrak{n}} .
\end{aligned}
$$

It is convenient to give a description of the $\mathcal{W}$-algebra as a subspace, rather than as a quotient space. Let $\ell^{\prime} \subset \mathfrak{g}_{\frac{1}{2}}$ be a maximal isotropic subspace (w.r.t. $\omega$ ) complementary to $\ell$. Hence, $\mathfrak{p}=\ell^{\prime} \oplus \mathfrak{g} \leq 0 \subset \mathfrak{g}$ is a subspace complementary to $\mathfrak{n}$ :

$$
\mathfrak{g}=\mathfrak{n} \oplus \mathfrak{p}=\mathfrak{n}^{\perp} \oplus \mathfrak{p}^{\perp} .
$$

(We can thus identify $\mathfrak{p}^{*}=\mathfrak{n}^{\perp}$ and $\mathfrak{n}^{*}=\mathfrak{p}^{\perp}$.) Consider the differential algebra homomorphism $\rho: \mathcal{V}_{z}(\mathfrak{g}) \rightarrow S(\mathbb{F}[\partial] \mathfrak{p})$ given by

$$
\rho(a)=\pi_{\mathfrak{p}}(a)+(f \mid a) \quad \forall a \in \mathfrak{g} .
$$

Clearly, $\operatorname{Ker}(\rho)=\langle n-(f \mid n)\rangle_{n \in \mathfrak{n}}$. Then, the classical $\mathcal{W}$-algebra can be equivalently be defined as follows:

$$
\mathcal{W}_{z}^{c l}(\mathfrak{g}, f) \simeq\left\{P \in S(\mathbb{F}[\partial] \mathfrak{p}) \mid \rho\left(\left\{a_{\lambda} P\right\}_{z}\right)=0 \forall a \in \mathfrak{n}\right\}
$$

with $\lambda$-bracket

$$
\left\{P_{\lambda} Q\right\}_{z, \rho}=\rho\left\{P_{\lambda} Q\right\}_{z}
$$

We can find explicit formulas for the $\lambda$-brackets of generators of $\mathcal{W}^{c l}(\mathfrak{g}, f)$, analogue to the result of Theorem 1.3.

Theorem 2.9 ([DSK13c]). As a differential algebra, the classical $\mathcal{W}$-algebra $\mathcal{W}_{z}^{c l}(\mathfrak{g}, f)$ is isomorphic to the algebra of differential polynomials over $\mathfrak{g}^{f}$, i.e. $\mathcal{W}_{z}^{c l}(\mathfrak{g}, f) \simeq$ $S\left(\mathbb{F}[\partial] \mathfrak{g}^{f}\right)$. Moreover, the $\lambda$ bracket on $\mathcal{W}_{z}^{\text {fin }}(\mathfrak{g}, f)$ is, using the notation in Theorem $1.3\left(q_{i_{0}}, q_{j_{0}} \in \mathfrak{g}^{f}\right)$ :

$$
\begin{aligned}
& \left\{p_{\lambda} q\right\}_{z}=\sum_{s, t=0}^{\infty} \sum_{i_{1}, \ldots, i_{s}=1}^{k} \sum_{j_{1}, \ldots, j_{t}=1}^{k} \sum_{m_{1}, \ldots, m_{s}=0}^{d} \sum_{n_{1}, \ldots, n_{t}=0}^{d} \\
& \left(\left[q_{j_{0}}, q_{n_{1}}^{j_{1}}\right]^{\sharp}-\delta_{n_{1}, 0} \delta_{j_{1}, j_{0}}(\lambda+\partial)\right) \ldots\left(\left[q_{j_{t-1}+1}^{n_{t-1}+1}, q_{n_{t}}^{j_{t}}\right]^{\sharp}-\delta_{n_{t}, n_{t-1}+1} \delta_{j_{t}, j_{t-1}}(\lambda+\partial)\right) \\
& \left(\left[q_{i_{s}}^{m_{s}+1}, q_{j_{t}}^{n_{t}+1}\right]^{\sharp}+\left(q_{i_{s}}^{m_{s}+1} \mid q_{j_{t}}^{n_{t}+1}\right)(\lambda+\partial)+z\left(s \mid\left[q_{i_{s}}^{m_{s}+1}, q_{j_{t}}^{n_{t}+1}\right]\right)\right. \\
& \left(\left[q_{i_{s-1}}^{m_{s-1}+1}, q_{m_{s}}^{i_{s}}\right]^{\sharp}+\delta_{m_{s}, m_{s-1}+1} \delta_{i_{s}, i_{s-1}}(\lambda+\partial)\right) \ldots\left(\left[q_{i_{0}}, q_{m_{1}}^{i_{1}}\right]^{\sharp}+\delta_{m_{1}, 0} \delta_{i_{1}, i_{0}} \lambda\right) .
\end{aligned}
$$

(In the RHS, when $s$ or $t$ is 0 , we replace $m_{0}+1$ or $n_{0}+1$ by 0 .)

In the special case classical $\mathcal{W}$-algebras for principal and minimal nilpotent element, equation (2.5) was proved in [DSKV13b].

2.4. Application of Poisson vertex algebras to the theory of Hamiltonian equations. Poisson vertex algebras can be used in the study of Hamiltonian partial differential equations in classical field theory, and their integrability [BDSK09] (in the same way as Poisson algebras are used to study Hamiltonian equations in classical mechanics).

The basic observation is that, if $\mathcal{V}$ is a Poisson vertex algebra with $\lambda$-bracket $\left\{\cdot{ }_{\lambda} \cdot\right\}$, then $\mathcal{V} / \partial \mathcal{V}$ is a Lie algebra, with Lie bracket

$$
\left\{\int f, \int g\right\}=\left.\int_{9}\left\{f_{\lambda} g\right\}\right|_{\lambda=0},
$$


and we have a representation of the Lie algebra $\mathcal{V} / \partial \mathcal{V}$ on $\mathcal{V}$, with the following action

$$
\left\{\int f, g\right\}=\left.\left\{f_{\lambda} g\right\}\right|_{\lambda=0},
$$

We can then introduce Hamiltonian equations and integrals of motion in the same way as in classical mechanics.

Definition 2.10. Given a Poisson vertex algebra $\mathcal{V}$ with $\lambda$-bracket $\left\{\cdot{ }_{\lambda} \cdot\right\}$, the Hamiltonian equation with Hamiltonian functional $\int h \in \mathcal{V} / \partial \mathcal{V}$ is:

$$
\frac{d u}{d t}=\left.\left\{h_{\lambda} u\right\}\right|_{\lambda=0}
$$

An integral of motion for the Hamiltonian equation (2.6) is an element $\int g \in \mathcal{V}$ such that

$$
\left\{\int h, \int g\right\}=\left.\int\left\{h_{\lambda} g\right\}\right|_{\lambda=0}=0 .
$$

The element $g \in \mathcal{V}$ is then called a conserved density. The usual requirement to have integrability is to have an infinite sequence $\int g_{0}=\int h, \int g_{1}, \int g_{2}, \ldots$ of linearly independent integrals of motion in involution:

$$
\left.\int\left\{g_{m_{\lambda}} g_{n}\right\}\right|_{\lambda=0}=0 \text { for all } m, n \in \mathbb{Z}_{+} .
$$

Example 2.11. The famous $K d V$ equation, describing the evolution of waves in shallow water is

$$
\frac{\partial u}{\partial t}=3 u \frac{\partial u}{\partial x}+c \frac{\partial^{3} u}{\partial x^{3}}
$$

It is a bi-Hamiltonian equation, since it can be written in two compatible Hamiltonian forms:

$$
\frac{d u}{d t}=\left.\left\{\frac{1}{2}\left(u^{3}+c u u^{\prime \prime}\right)_{\lambda} u\right\}_{0}\right|_{\lambda=0}=\left.\left\{\frac{1}{2} u^{2}{ }_{\lambda} u\right\}_{1}\right|_{\lambda=0},
$$

on the differential algebra $\mathcal{V}=S(\mathbb{F}[\partial] u)$, with PVA $\lambda$-brackets

$$
\left\{u_{\lambda} u\right\}_{0}=\lambda,\left\{u_{\lambda} u\right\}_{1}=u^{\prime}+2 u \lambda+c \lambda^{3} .
$$

Compatibility means that $\left\{\cdot{ }_{\lambda} \cdot\right\}_{z}=\left\{\cdot{ }_{\lambda} \cdot\right\}_{0}+\left\{\cdot{ }_{\lambda} \cdot\right\}_{1}$ is a 1-parameter family of PVA $\lambda$-brackets.

The usual "trick" to construct a sequence $\int g_{n}, n \in \mathbb{Z}_{+}$, of integrals of motion in involution is the so called Lenard-Magri scheme: assuming we have a biHamiltonian equation

$$
\frac{d u}{d t}=\left\{h_{1 \lambda} u\right\}_{0}=\left\{h_{0 \lambda} u\right\}_{1},
$$

we try to solve the following recursion equation for $\int g_{n}, n \geq 0$ (starting with $g_{0}=h_{0}$ and $\left.g_{1}=h_{1}\right)$,

$$
\left\{g_{0 \lambda} u\right\}_{0}=0, \quad\left\{g_{n+1 \lambda} u\right\}_{0}=\left\{g_{n_{\lambda}} u\right\}_{1} .
$$

(There are various "cohomologcal" arguments indicating that, often, such recursive equations can be solved for every $n$, see e.g. [DSK12a, DSK12b].) In this case, it was a simple observation of Magri [Mag78] that the solutions $\int g_{n}, n \in \mathbb{Z}_{+}$, are integrals of motion in involution w.r.t. both PVA $\lambda$-brackets $\left\{\cdot{ }_{\lambda} \cdot\right\}_{0}$ and $\left\{\cdot{ }_{\lambda} \cdot\right\}_{1}$, and therefore we get the integrable hierarchy of bi-Hamiltonian equations

$$
\frac{d u}{d t_{n}}=\left\{g_{n_{\lambda}} u\right\}_{0} .
$$


2.5. Generalized Drinfeld-Sokolov bi-Hamiltonian integrable hierarchies. Following the ideas of [DS85], we can prove that the Lenard-Magri scheme can be applied to construct integrable hierarchies of bi-Hamiltonian equations attached to the classical $\mathcal{W}$-algebras $\mathcal{W}_{z}^{c l, f i n}(\mathfrak{g}, f)$. For example, for $\mathfrak{g}=\mathfrak{s l}_{2}$, we get the $\mathrm{KdV}$ hierarchy (cf. Example 2.11).

The basic assumption is that there exists a homogeneous (w.r.t. the ad $x$ eigenspace decomposition) element $s \in \operatorname{Ker}(\operatorname{ad} \mathfrak{n})$ such that $f+s$ is a semisimple element of $\mathfrak{g}$. Hence, $f+z s$ is a semisimple element of $\mathfrak{g}\left(\left(z^{-1}\right)\right)$, and we have the direct sum decomposition $\mathfrak{g}\left(\left(z^{-1}\right)\right)=\mathfrak{h} \oplus \mathfrak{h}^{\perp}$, where

$$
\mathfrak{h}:=\operatorname{Ker} \operatorname{ad}(f+z s) \text { and } \mathfrak{h}^{\perp}:=\operatorname{Imad}(f+z s) .
$$

We define a $\frac{1}{2} \mathbb{Z}$-grading of $\mathfrak{g}\left(\left(z^{-1}\right)\right)$ by letting $\operatorname{deg}(z)=-d-1$, if $s \in \mathfrak{g}_{d}$. In particular, $f+z s$ is homogenous of degree -1 . We have the induced decompositions of $\mathfrak{h}$ and $\mathfrak{h}^{\perp}$ (since $f+z s$ is homogeneous):

$$
\mathfrak{h}=\widehat{\bigoplus}_{i \in \frac{1}{2} \mathbb{Z}} \mathfrak{h}_{i} \text { and } \mathfrak{h}^{\perp}=\widehat{\bigoplus}_{i \in \frac{1}{2} \mathbb{Z}} \mathfrak{h}_{i}^{\perp}
$$

Consider the Lie algebra

$$
\tilde{\mathfrak{g}}=\mathbb{F} \partial \ltimes\left(\mathfrak{g}\left(\left(z^{-1}\right)\right) \otimes \mathcal{V}(\mathfrak{p})\right),
$$

where $\partial$ acts only on the second factor of the tensor product. Clearly, $\mathfrak{g}\left(\left(z^{-1}\right)\right)_{>0} \otimes$ $\mathcal{V}(\mathfrak{p}) \subset \tilde{\mathfrak{g}}$ is a pro-nilpotent subalgebra. Hence, for $U(z) \in \mathfrak{g}\left(\left(z^{-1}\right)\right)_{>0} \otimes \mathcal{V}(\mathfrak{p})$, we have a well defined automorphism $e^{\operatorname{ad} U(z)}$ of $\tilde{\mathfrak{g}}$. Let $\left\{q_{i}\right\}_{i \in J}$ be a basis of $\mathfrak{p}$, and let $\left\{q^{i}\right\}_{i \in J}$ be the dual basis of $\mathfrak{n}^{\perp}$.

Theorem 2.12 ([DSKV13a]). (a) There exist unique $U(z) \in \mathfrak{h}_{>0}^{\perp} \otimes \mathcal{V}(\mathfrak{p})$ and $h(z) \in$ $\mathfrak{h}_{>-1} \otimes \mathcal{V}(\mathfrak{p})$ such that

$$
e^{\operatorname{ad} U(z)}\left(\partial+(f+z s) \otimes 1+\sum_{i \in J} q^{i} \otimes q_{i}\right)=\partial+(f+z s) \otimes 1+h(z) .
$$

(b) For $0 \neq a(z) \in Z(\mathfrak{h})$, the coefficients $g_{n}, n \in \mathbb{Z}_{+}$, of the Laurent series

$$
g(z)=(a(z) \otimes 1 \mid h(z)) \in \mathcal{V}(\mathfrak{p})\left(\left(z^{-1}\right)\right),
$$

lie in $\mathcal{W}_{z}^{c l, f i n}(\mathfrak{g}, f) \subset \mathcal{V}(\mathfrak{p})$ modulo $\partial \mathcal{V}(\mathfrak{p})$, and they satisfy the Lenard-Magri recursion equations (2.7) for the PVA $\lambda$-brackets $\left\{\cdot{ }_{\lambda} \cdot\right\}_{0}=\left\{\cdot{ }_{\lambda} \cdot\right\}_{z=0}$ and $\left\{\cdot{ }_{\lambda} \cdot\right\}_{1}=$ $\left.\frac{d}{d z}\{\cdot \lambda \cdot\}_{z}\right|_{z=0}$

Hence, we get an integrable hierarchy of bi-Hamiltonian equations, called the generalized Drinfeld-Sokolov hierarchy $(w \in \mathcal{W})$,

$$
\frac{d w}{d t_{n}}=\left.\rho\left\{g_{n_{\lambda}} w\right\}_{0}\right|_{\lambda=0}, \quad n \in \mathbb{Z}_{+}
$$

\section{REFERENCES}

[Ara07] Arakawa T., Representation theory of W-algebras, Invent. Math. 169 (2007), no. 2, 219320.

[BDSK09] Barakat A., De Sole A., Kac V., Poisson vertex algebras in the theory of Hamiltonian equations, Jpn. J. Math. 4, (2009), no. 2, 141-252.

[Bor86] Borcherds R., Vertex algebras, Kac-Moody algebras and the Monster, Proc. Natl. Acad. Sci. USA 83 (1986), 3068-3071.

[BdGHM93] Burruoughs N., de Groot M., Hollowood T., Miramontes L., Generalized DrinfeldSokolov hierarchies II: the Hamiltonian structures, Comm. Math. Phys. 153 (1993), 187-215.

[BS95] Bouwknegt P. , Schoutens K., W-symmetry, Advanced ser. in Math. Phys. 22, World Sci., 1995.

[BrK06] Brundan J., Kleshchev A., Shifted Yangians and finite W-algebras Adv. Math. 200 (2006), no.1, 136-195.

[CM93] Collingwood D., McGovern W., Nilpotent orbits in semisimple Lie algebras, Van Nostrand Reinhold Mathematics Series, Van Nostrand Reinhold Co., New York, 1993. 
[DF95] Delduc F., Fehér L., Regular conjugacy classes in the Weyl group and integrable hierarchies, J. Phys. A 28 (1995), no. 20, 5843-5882 .

[dGHM92] de Groot M., Hollowood T., Miramontes L., Generalized Drinfeld-Sokolov hierarchies, Comm. Math. Phys. 145 (1992), 57-84.

[DSK06] De Sole A., Kac V.G., Finite vs. affine W-algebras, Jpn. J. Math. 1 (2006), no.1, 137-261.

[DSK12a] De Sole A., Kac V.G., The variational Poisson cohomology, Jpn. J. Math. 8 (2013), no.1, 1-145.

[DSK12b] De Sole A., Kac V.G., Essential variational Poisson cohomology, Comm. Math. Phys. 313 (2012), no.3, 837-864.

[DSK06] De Sole A., Kac V.G., Finite vs. affine W-algebras, Jpn. J. Math. 1 (2006), no. 1, $137-261$.

[DSKV13a] De Sole A., Kac V.G., Valeri D., Classical $\mathcal{W}$-algebras and generalized DrinfeldSokolov bi-Hamiltonian systems within the theory of Poisson vertex algebras, to appear in Communications in Mathematical Physics, arXiv:1207.6286v3

[DSKV13b] De Sole A., Kac V.G., Valeri D., Classical W-algebras and generalized DrinfeldSokolov hierarchies for minimal and short nilpotents, preprint arXiv:1306.1684

[DSK13c] De Sole A., Kac V.G., W-algebras, in preparation.

[DS85] Drinfeld V.G., Sokolov V.V., Lie algebras and equations of Korteweg-de Vries type, Soviet J. Math. 30 (1985), 1975-2036.

[FHM92] Fehér L., Harnad J., Marshall I., Generalized Drinfeld-Sokolov reductions and KdV type hierarchies, Comm. Math. Phys. 154 (1993), no. 1, 181-214.

[FF90] Feigin B.L., Frenkel, E., Quantization of Drinfeld-Sokolov reduction, Phys. Lett., B 246 (1990), 75-81.

[FF90b] Feigin B.L., Frenkel E., Affine Kac-Moody algebras, bosonization and resolutions, Lett. Math. Phys. 19 (1990), 307-317.

[FGMS95] Fernández-Pousa C., Gallas M., Miramontes L., Sánchez Guillén J., W-algebras from soliton equations and Heisenberg subalgebras. Ann. Physics 243 (1995), no. 2, 372-419.

[FGMS96] Fernández-Pousa C., Gallas M., Miramontes L., Sánchez Guillén J., Integrable systems and $\mathcal{W}$-algebras, VIII J. A. Swieca Summer School on Particles and Fields (Rio de Janeiro, 1995), 475-479.

[GG02] Gan, W.L., Ginzburg V., Quantization of Slodowy slices, Int. Math. Res. Not. 5 (2002), 243-255

[GS90] Guillemin V., Sternberg S., Symplectic techniques in physics, Second edition. Cambridge University Press, Cambridge, 1990.

[KRW03] Kac V.G., Roan S.-S., Wakimoto M., Quantum reduction for affine superalgebras, Comm. Math. Phys., 241 (2003), 307-342.

[KW04] Kac V. G., Wakimoto M., Quantum reduction and representation theory of superconformal algebras, Adv. Math. 185 (2004), 400-458. Corrigendum, Adv. Math. 193 (2005), 453-455.

[KW05] Kac V. G., Wakimoto M., Quantum reduction in the twisted case, in Progress in Math. 237 (2005), pp 85-126.

[Kos78] Kostant B., On Whittaker vectors and representation theory, Inv. Math 48 (1978), 101184.

[Lyn79] Lynch, T.E., Generalized Whittaker vectors and representation theory, Thesis (Ph.D.) Massachusetts Institute of Technology, 1979.

[Mag78] Magri F., A simple model of the integrable Hamiltonian equation, J. Math. Phys. 19 (1978), no.5, 1156-1162.

[Mat90] Matumoto H., Whittaker modules associated with highest weight modules, Duke Math. J. 60 (1990), 59-113.

[Pre02] Premet A., Special transverse slices and their enveloping algebras, Adv. Math. 170 (2002), $1-55$.

[Pre05] Premet A., Enveloping algebras of Slodowy slices and the Joseph ideal, J. Eur. Math. Soc. (JEMS) 9 (2007), no.3, 487-543.

[Slo80] Slodowy P., Simple singularities and simple algebraic groups, Lecture Notes in Mathematics, 815. Springer, Berlin, 1980.

[Va94] Vaisman I., Lectures on the geometry of Poisson manifolds, Progress in Mathematics, 118 Birkhäuser Verlag, Basel, 1994.

[Zam85] Zamolodchikov A., Infinite extra symmetries in two-dimensional conformal quantum field theory, Teor. Mat. Fiz 65(3)(1985),347-359.

[Zhu96] Zhu Y., Modular invariance of characters of vertex operator algebras, Journal AMS 9 (1996), 237-302. 